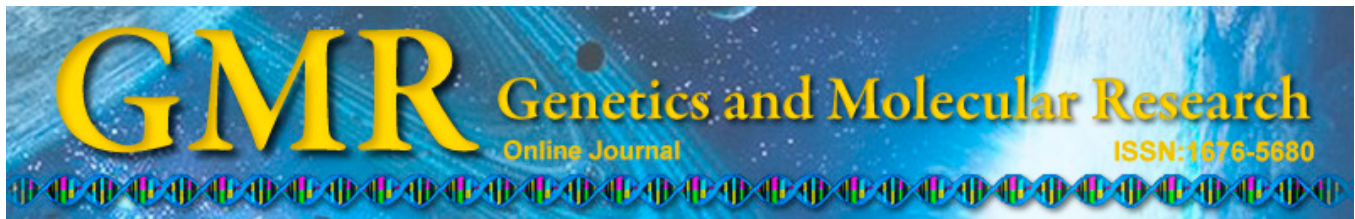

\title{
Preoperative computed tomography-guided hook-wire positioning of pulmonary nodules
}

\author{
W. Li' ${ }^{1}$, C.B. Zhai', Y.J. Guan' ${ }^{2}$, L.J. Jing ${ }^{2}$, Z.L. Zhang ${ }^{2}$ and X.H. Zhao ${ }^{2}$ \\ 'Department of Thoracic Surgery, Weifang People's Hospital, Weifang, \\ Shandong, China \\ ${ }^{2}$ Department of Thoracic Surgery, \\ Affiliated Hospital of Weifang Medical University, Weifang, Shandong, China \\ Corresponding author: X.H. Zhao \\ E-mail: zhaoxhdts@126.com
}

Genet. Mol. Res. 14 (2): 3798-3806 (2015)

Received July 10, 2014

Accepted November 13, 2014

Published April 22, 2015

DOI http://dx.doi.org/10.4238/2015.April.22.9

\begin{abstract}
This study aimed to analyze the clinical application value of computed tomography (CT)-guided hook-wire positioning before thoracoscopic surgery. Eighty-four patients who had received a thoracoscopic wedge resection of pulmonary nodules between January and December 2013 were selected. Group A consisted of 38 cases where the hook-wire positioning technique was not used, and the positioning approaches were intraoperative observation and palpation. Group B consisted of 46 cases where the hook-wire positioning technique was used. The diameter of each nodule was less than $2 \mathrm{~cm}$, and all patients underwent the operation within $2 \mathrm{~h}$ of invasive positioning. The evaluation indexes included positioning success rate, positioningrelated complications, and rate of conversion to thoracotomy. In Group $\mathrm{A}$, nine patients (23.68\%) underwent conversion to thoracotomy; in Group B, three patients $(6.52 \%)$ did. This difference was statistically significant $(\mathrm{P}<0.05)$. The average operation duration was $118 \pm 21$ min in Group A and $53 \pm 18$ min in Group B. The difference between both groups was statistically significant $(\mathrm{P}<0.05)$. The average length
\end{abstract}


of hospital stay of patients who underwent conversion to thoracotomy was $8.7 \pm 2.2$ days, and of patients who underwent thoracoscopic pulmonary wedge resection was $4.5 \pm 1.6$ days. This difference was statistically significant $(\mathrm{P}<0.05)$. Therefore, CT-guided hook-wire positioning of pulmonary nodules before thoracoscopic surgery has clinical application value. It helps to accurately locate the pulmonary nodules, effectively lowers the rate of conversion to thoracotomy, and reduces the operation duration.

Key words: Computed tomography guided positioning; Hook-wire; Pulmonary nodules; Thoracoscopic surgery

\section{INTRODUCTION}

As computed tomography (CT) has become widely applied in physical examinations and follow-up visits in tumor patients, more and more pulmonary nodules have been found. However, a pathological diagnosis is still currently the golden criterion for diagnosis (Furman et al., 2013). Considering that pulmonary nodules with a diameter greater than $5 \mathrm{~mm}$ have a very high risk of malignancy, it is necessary to use invasive approaches to obtain a pathological diagnosis. CT-guided percutaneous pulmonary puncture biopsy and fiber bronchoscope biopsy are common methods used to obtain histological samples of pulmonary nodules (Lee et al., 2012). However, for pulmonary nodules with a diameter less than $1.5 \mathrm{~cm}$ the accuracy rate of puncture biopsy decreases significantly. Relevant literature has reported that the possibility of malignancy cannot be excluded in $29 \%$ of these nodules, due to a lack of histological evidence (Henschke et al., 2011). Therefore, for pulmonary nodules with a diameter less than $1 \mathrm{~cm}$, the thoracoscope becomes the unique choice for obtaining histological samples (Shan et al., 2013). It has been reported that the hook-wire positioning technique has many complications, particularly unhooking (Andrade, 2010; Ambrogi et al., 2012). In addition, partial reports have indicated that the cutting closer has cut off the steel wires during surgery (Huang et al., 2014).

This research aims to evaluate the clinical application value of CT-guided positioning before thoracoscopic surgery for pulmonary nodules using outcome parameters such as the success rate of positioning, the rate of conversion to thoracotomy, complications, and accidents.

\section{MATERIAL AND METHODS}

\section{Material and equipment}

The hook-wire was purchased from BARD, New Jersey, USA; the CT apparatus (Somatom Sensation 32) was purchased from Siemens, Erlangen, Germany; the positioning bed was purchased from Huanxi Medical Apparatus and Instruments Co., Ltd., Shanghai, China; the disposable cutting closer was purchased from Jinning Medical Apparatus and Instruments Co., Ltd., Hefei, China.

\section{Selection of cases}

All patients included in the research underwent a thoracoscopic wedge resection, but 
any with a lump diameter greater than $2 \mathrm{~cm}$ or a distance between the lump and the pulmonary pleura greater than $35 \mathrm{~mm}$ were excluded (De Boo et al., 2011).

Between January and December 2013, 84 patients (51 men and 33 women) with pulmonary nodules less than $2 \mathrm{~cm}$ in diameter underwent thoracoscopic pulmonary wedge resection in our hospital. With an average age of $57.3 \pm 12.6$ years (range, 26-72 years), all patients were subject to thoracic CT examinations. Based on measurements from CT images, the diameter of all nodules was less than $2 \mathrm{~cm}$ and the average distance between the nodule and the pulmonary pleura was $16 \pm 9 \mathrm{~mm}$ (range, $0-35 \mathrm{~mm}$ ). No definite diagnosis was made for any of the patients before surgery.

\section{Experimental grouping}

Group A consisted of 38 patients and Group B of 46 patients. Group A received thoracoscopic pulmonary wedge resection, with positioning carried out using intraoperative observation and palpation rather than the hook-wire positioning technique. Group B received thoracoscopic pulmonary wedge resection with preoperative hook-wire positioning.

Each patient had only one pulmonary nodule, and the preoperative CT-guided puncture for all patients was performed by the same doctor. Records were made of the diameter of pulmonary nodules, the distance between the pulmonary nodule and the pleura, and the duration of puncture operation (from administration of local anesthesia to cutting the hook-wire at the level of skin). Records were made of the patients' final pathology results, the success rate of positioning, the ratio of conversion to thoracotomy, the duration of thoracoscopic surgery, postoperative complications, intraoperative accidents, and the average length of hospital stay in days.

\section{Positioning method}

On the day of surgery, the patient was transferred to the CT room $2 \mathrm{~h}$ before the operation, and the patient and his/her family members signed an informed consent form. The shortest path necessary for puncture determined the patient posture. Three-dimensional coordinates were established using the patients' body surface signs, three-dimensional reconstruction, and the CT puncture biopsy scanning sequence. The needle insertion angle and distance were accurately marked using three-dimensional coordinates, and the puncture points and needle insertion passage were defined using laser positioning lines and body surface signs. After local sterilization and anesthesia, the puncture needle was threaded through the nodule as much as possible, or threaded through the pulmonary tissue most adjacent to the nodule if the nodule was excessively small (Liu et al., 2013). The hook-wire was released when the trocar needle reached a satisfactory position. A CT reexamination was performed to confirm the position of the hook-wire after the trocar needle was withdrawn. The metal wire was cut off at the level of the skin, and the patient was transferred to the operation room for surgery if the position was satisfactory (Figure 1).

\section{Operation method}

Routine double-lumen trachea cannulation and one-lung ventilation were performed for the operation. Pulmonary lobectomy and lymph node dissection were required to be performed under an endoscope because pulmonary nodules might be pathologically malignant. 
Therefore, the observation incision was located at the 7 th rib in the midaxillary line, the main operating incision was located at the 4th rib in the anterior axillary line, and the axillary operating incision was located at the 9 th rib. Wedge resections of lumps were performed using a disposable cutting closer. All removed tissue was placed in specimen bags and removed from the body to avoid the possibility of tumor implantation. The specimens were frozen. Pulmonary lobectomy and lymph node dissection were performed under an endoscope if the pathology was primary pulmonary non-small-cell carcinoma (Yoshida et al., 2011).
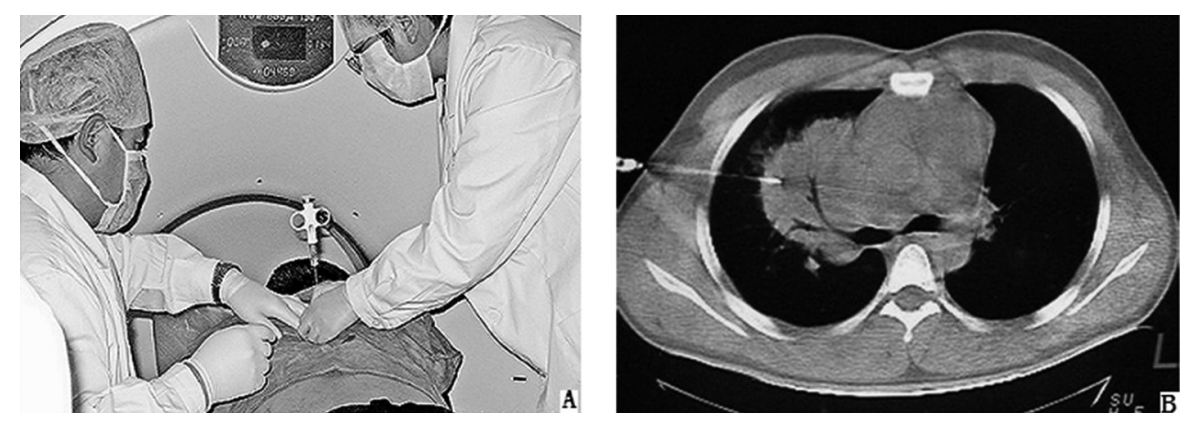

Figure 1. A. Computed tomography (CT)-guided percutaneous pulmonary puncture. B. CT image following percutaneous pulmonary puncture.

\section{Statistical methods}

The data are reported as means \pm standard deviation. The chi-square test was used for comparison between the groups. For the multivariate analysis, the logistic regression analysis method was used to evaluate the effect of factors such as lump size and distance from the pleura on the rate of conversion to thoracotomy.

\section{RESULTS}

\section{Characteristics of pulmonary nodules}

There were 29 patients in Group A and 21 patients in Group B with a distance between the pulmonary nodule and the pulmonary pleura of less than $1.5 \mathrm{~cm}$. There were 15 patients in Group A and 27 patients in Group B with pulmonary nodules with a diameter less than $1 \mathrm{~cm}$. Table 1 shows the specific characteristics of the pulmonary nodules.

\begin{tabular}{lcc} 
Table 1. Characteristics of pulmonary nodules. & & \\
\hline & Group A & Group B \\
\hline Site & & 28 \\
$\quad$ Upper middle lobe & 22 & 18 \\
$\quad$ Lower lobe & 16 & 27 \\
Diameter & 15 & 19 \\
$\quad \leq 1 \mathrm{~cm}$ & 23 & 21 \\
$\quad>1 \mathrm{~cm}$ & 29 & 25 \\
Distance frompleura & 29 & \\
$\quad 1.5 \mathrm{~cm}$ & 9 & $\mathrm{~cm}$
\end{tabular}




\section{Causes of conversion to thoracotomy}

Conversion to thoracotomy was required in nine patients in Group A (23.68\%). The primary causes of conversion to thoracotomy were failure of positioning by finger palpation (six cases), and severe pleural adhesions (three cases). The diameter of the pulmonary nodules of the six patients with positioning failure was less than $1 \mathrm{~cm}$. Conversion to thoracotomy was required in three patients in Group B (6.52\%). One patient had a lump that was too small after unhooking, and positioning by palpation was not possible. The other two patients had severe pleural adhesions. Therefore, the rates of conversion to thoracotomy in Groups A and B were 23.68 and $6.52 \%$, respectively, and the difference between these was statistically significant ( $\mathrm{P}$ $<0.05)$. Table 2 shows the specific causes of conversion.

Table 2. Causes of conversion to thoracotomy.

\begin{tabular}{lcc}
\hline & Group A & Group B \\
\hline Preoperative puncture failure & 0 & 0 \\
Unhooking & 0 & 1 \\
Intraoperative positioning failure & 6 & 0 \\
Severe pleural adhesions & 3 & 2 \\
Total (\%) & $9(23.68 \%)$ & $3(6.52 \%)$ \\
\hline
\end{tabular}

\section{Pathology results of pulmonary nodules}

Definite pathological diagnoses were determined in all patients. Malignant nodules were diagnosed in 53 cases ( 24 cases in Group A and 29 cases in Group B). Primary lung cancer was diagnosed in 34 cases (15 cases in Group A and 19 cases in Group B) and metastatic cancer was diagnosed in 19 cases ( 9 cases in Group A and 10 cases in Group B). Other diseases were diagnosed in 31 patients $(\mathrm{P}>0.05)$. Figure 2 shows the specific pathology results.

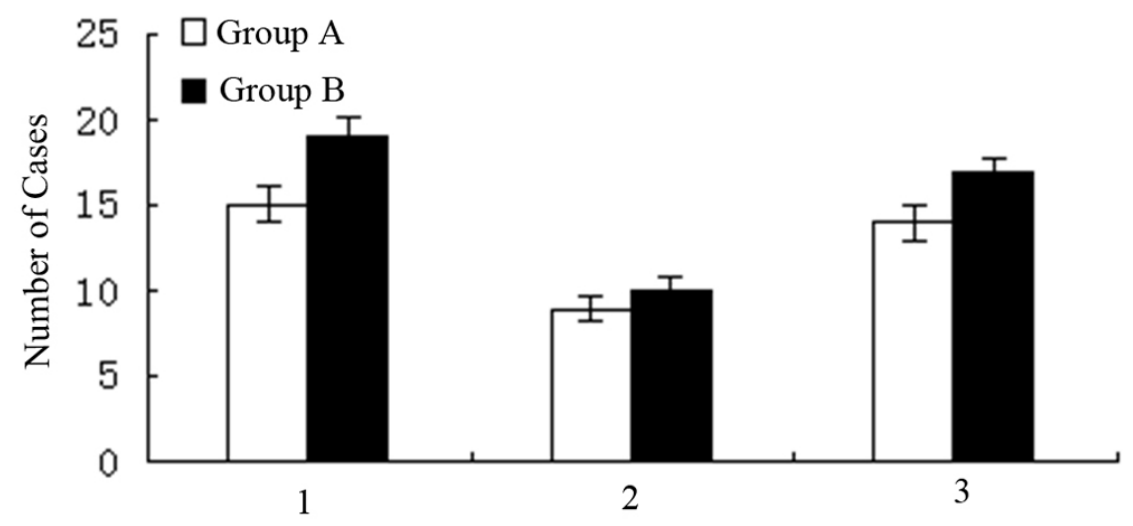

Figure 2. Pathology results of pulmonary nodules. 1: primary lung cancer; 2: metastatic cancer; 3: other diseases.

\section{Intraoperative conditions}

The surgery was successfully performed for all patients in Group A. Accidents oc- 
curred with three patients in Group B. These accidents occurred because the nodule position was deep and the positioning steel wire placement was excessively deep. For two of the three patients, the positioning steel wire was cut off by the cutting closer during removal and remained in the healthy lung. It was then immediately removed endoscopically. In the third case, the steel wire was deformed by the head of the cutting closer instead of being cut off, and pulmonary wedge resections were completed under the direct guidance of the hook-wire (see Figure 3).
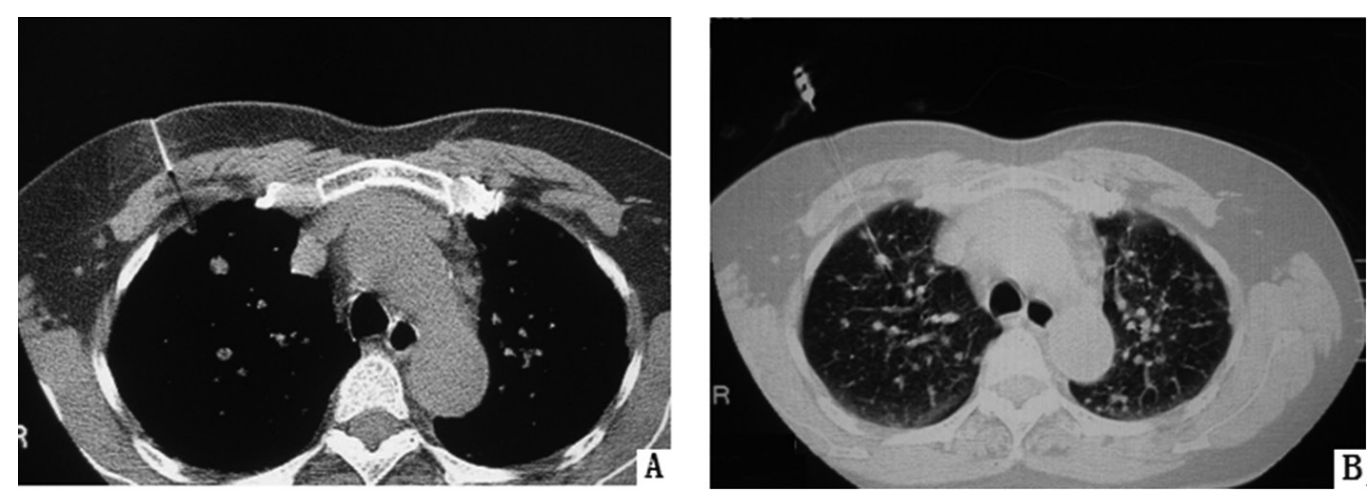

Figure 3. Computed tomography (CT) images showing the results of intraoperative accidents. A. The positioning steel wire was cut off by the cutting closer and remained in the lung due to excessively deep placement. B. The steel wire was deformed by the head of the cutting closer instead of being cut off.

\section{Operation results}

The average operation duration was $118 \pm 21 \mathrm{~min}$ in Group A and $53 \pm 18 \mathrm{~min}$ in Group B. The difference between the groups was statistically significant $(\mathrm{P}<0.05$; see Figure $4 \mathrm{~A})$. The average length of hospital stay of patients who underwent conversion to thoracotomy was $8.7 \pm 2.2$ days and the average length of hospital stay of patients who underwent thoracoscopic pulmonary wedge resection was $4.5 \pm 1.6$ days. The difference in length of stay between these two types of patients was statistically significant $(P<0.05$; see Figure 4B).

A

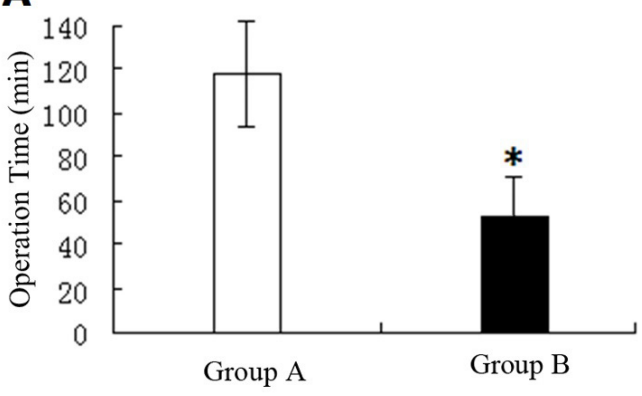

B

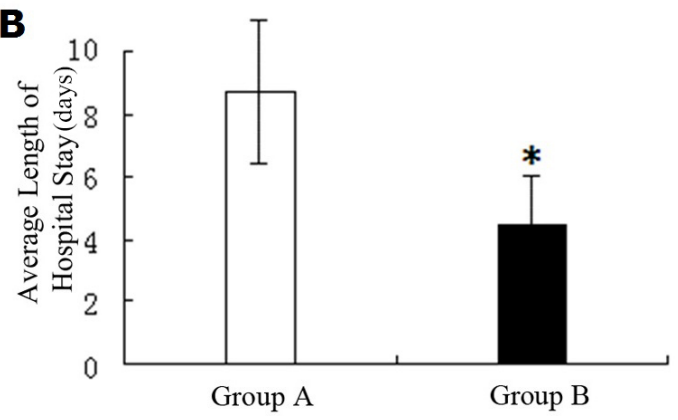

Figure 4. A. Average operation duration in Group A and in Group B. B. Average length of hospital stay of patients who underwent conversion to thoracotomy and who underwent thoracoscopic pulmonary wedge resection. ${ }^{*} \mathrm{P}<0.05$. 


\section{DISCUSSION}

In recent years, with the development of minimally invasive thoracic surgery, thoracoscopic surgery has been increasingly accepted by a wide range of patients and medical care personnel. To solve the problem of pulmonary nodule positioning, most doctors still use intraoperative finger palpation. The main advantage of palpation lies in the fact that the technique is a very simple method for both doctors and patients (Hwang et al., 2010). For nodules less than $1 \mathrm{~cm}$ deep to the pleura and with a diameter greater than $1 \mathrm{~cm}$, the method is very effective (Yin et al., 2012). The finger palpation positioning technique is safe and incurs no extra expense, but the technique relies on the doctor's experience and has some obvious defects. It is not possible to distinguish nodules with a diameter less than $1 \mathrm{~cm}$, those far away from the pleura in the visceral layer, and soft lumps. In addition, it is not possible to observe the position of nodules from the changes in the pulmonary surface profile, even if the lung collapses completely (Oda et al., 2010; Prosch et al., 2011). It is not possible to accurately determine the position of some superficial tiny pulmonary nodules (with a diameter less than $5 \mathrm{~mm}$ ), which leads to conversion to thoracotomy and missed diagnoses (Calcagni et al., 2012). To solve the problem, many methods of preoperative or intraoperative positioning are used clinically, e.g., hook-wire positioning, methylene blue staining, fluoroscopy assisted positioning, and the use of microscopic tactile sensors, intraoperative CT, finger palpation, intraoperative ultrasound, radio guidance and agar marking (Cao et al., 2012; Takeda et al., 2012).

Hook-wire positioning is one of the preoperative invasive positioning techniques. All of the invasive positioning techniques carry a risk of complications such as pneumothorax, chest pain, pulmonary hemorrhage, and cough, because pulmonary puncture needs to be performed (Ichinose et al., 2013; Nouri et al., 2013). It has been reported that the hook-wire positioning technique has multiple complications, particularly unhooking. Unhooking frequently occurs during patient transfer or during surgery, and may occur under the state of pulmonary collapse or during excision because the operator often strains the positioning steel wire when excising lumps (Tsushima et al., 2008; Masroor et al., 2012). In addition, partial reports have indicated that the cutting closer may cut off the steel wire during surgery (Rozen et al., 2010). Designing a rational angle of needle insertion is critical in ensuring successful positioning of small focuses (Kawanaka et al., 2009). In this study, three-dimensional coordinates were established using the patients' body surface signs, three-dimensional reconstruction, and the CT puncture biopsy scanning sequence. The needle insertion angle and distance were accurately marked using three-dimensional coordinates, and the puncture points and needle insertion passage were defined using laser positioning lines and body surface signs. Increasing contact with the pulmonary tissue is a prerequisite in preventing dislocation of a metal hook (Nakamoto et al., 2010; Slotty and Stummer, 2012). In our study, the metal hook penetrated deep through the nodules into the pulmonary tissue below $(1-2 \mathrm{~cm})$, increasing the contact area of the metal hook. The metal wire outside of the body surface was cut off immediately after positioning to reduce traction from external forces.

For some pulmonary nodules in specific positions, trapezoid or obtuse triangle resection may be performed instead of the traditional wedge resection, due to selection of the position of the operating incision during thoracoscopic pulmonary wedge resection (Omori et al., 2010; Tamura et al., 2010). In addition, it is difficult to perform deep wedge resections at sites on the truncated edge of the lung. Hence, hook-wire positioning was performed for pulmonary nodules on the truncated edge of the lung (e.g., posterior section of the lower lobe) 
at a depth of more than $2 \mathrm{~cm}$. The thoracoscopic wedge resection has many risks, e.g., positive incisal margins, removal of excessive pulmonary tissue, failure to guarantee a rational distance between the incisal margins and the nodules, and retention of foreign matter due to cutting of the steel wire (Iguchi et al., 2009; Magistrelli et al., 2009). Thus, care should be taken in performing a wedge resection for deep pulmonary nodules, and preparations should be made for the resection of pulmonary segments or lobes (Miyoshi et al., 2009). In cases where removal of a pulmonary segment or lobe is required following resection failure or retention of foreign matter, high-resolution thin-layer CT scanning is used to define the pulmonary segment where the nodule is located.

In conclusion, thoracoscopic preoperative CT-guided hook-wire positioning is a quick, accurate, and effective method that contributes to the removal of pulmonary nodules that are small in diameter and soft in texture. It can effectively reduce the rate of conversion to thoracotomy, decrease the operation time, and expand the surgical indications. The technique has some imperfections in positioning superficial tiny pulmonary nodules and deep pulmonary nodules. The number of cases used in our study was small, and the sample size needs to be expanded in future research in order to obtain more credible results. The technique used in this study has not been widely applied in China, so this research aims to increase awareness of it there.

\section{REFERENCES}

Ambrogi MC, Melfi F, Zirafa C, Lucchi M, et al. (2012). Radio-guided thoracoscopic surgery (RGTS) of small pulmonary nodules. Surg. Endosc. 26: 914-919.

Andrade RS (2010). Electromagnetic navigation bronchoscopy-guided thoracoscopic wedge resection of small pulmonary nodules. Semin. Thorac. Cardiovasc. Surg. 22: 262-265.

Calcagni ML, Taralli S, Maggi F, Rufini V, et al. (2012). ${ }^{18} \mathrm{~F}$-fluoro-deoxy-glucose focal uptake in very small pulmonary nodules: fact or artifact? Case reports. World J. Surg. Oncol. 10: 71.

Cao JQ, Rodrigues GB, Louie AV and Zaric GS (2012). Systematic review of the cost-effectiveness of positron-emission tomography in staging of non-small-cell lung cancer and management of solitary pulmonary nodules. Clin. Lung Cancer 13: 161-170.

De Boo DW, Uffmann M, Weber M, Bipat S, et al. (2011). Computer-aided detection of small pulmonary nodules in chest radiographs: an observer study. Acad. Radiol. 18: 1507-1514.

Furman AM, Dit Yafawi JZ and Soubani AO (2013). An update on the evaluation and management of small pulmonary nodules. Future Oncol. 9: 855-865.

Henschke CI, Yankelevitz DF, Reeves AP and Cham MD (2011). Image analysis of small pulmonary nodules identified by computed tomography. Mt. Sinai J. Med. 78: 882-893.

Huang W, Ye H, Wu Y, Xu W, et al. (2014). Hook wire localization of pulmonary pure ground-glass opacities for videoassisted thoracoscopic surgery. Thorac. Cardiovasc. Surg. 62: 174-178.

Hwang HS, Chung MJ, Lee JW, Shin SW, et al. (2010). C-arm cone-beam CT-guided percutaneous transthoracic lung biopsy: usefulness in evaluation of small pulmonary nodules. AJR Am. J. Roentgenol. 195: 400-407.

Ichinose J, Kohno T, Fujimori S, Harano T, et al. (2013). Efficacy and complications of computed tomography-guided hook wire localization. Ann. Thorac. Surg. 96: 1203-1208.

Iguchi T, Yoshioka T, Muro M, Miyasho K, et al. (2009). Systemic air embolism during preoperative pulmonary marking with a short hook wire and suture system under CT fluoroscopy guidance. Jpn. J. Radiol. 27: 385-388.

Kawanaka K, Nomori H, Mori T, Ikeda K, et al. (2009). Marking of small pulmonary nodules before thoracoscopic resection: injection of lipiodol under CT-fluoroscopic guidance. Acad. Radiol. 16: 39-45.

Lee HK, Cho SW, Lee HS, Kim KI, et al. (2012). Comparing 18F-Fluorodeoxyglucose Positron Emission Tomography and Video-assisted Thoracoscopic Surgery in the Evaluation of Small Pulmonary Nodules in Patients with a History of Malignancy. Korean J. Thorac. Cardiovasc. Surg. 45: 35-39.

Liu M, Lv Y, Wu L, Song J, et al. (2013). MRI-guided percutaneous coaxial cutting needle biopsy of small pulmonary nodules: feasibility. Eur. Radiol. 23: 2730-2738.

Magistrelli P, D’Ambra L, Berti S, Feleppa C, et al. (2009). Use of India ink during preoperative computed tomography 
localization of small peripheral undiagnosed pulmonary nodules for thoracoscopic resection. World J. Surg. 33: 1421-1424.

Masroor I, Afzal S, Shafqat G and Rehman H (2012). Usefulness of hook wire localization biopsy under imaging guidance for nonpalpable breast lesions detected radiologically. Int. J. Womens Health 4: 445-449.

Miyoshi K, Toyooka S, Gobara H, Oto T, et al. (2009). Clinical outcomes of short hook wire and suture marking system in thoracoscopic resection for pulmonary nodules. Eur. J. Cardiothorac. Surg. 36: 378-382.

Nakamoto K, Omori K and Nezu K (2010). Superselective segmentectomy for deep and small pulmonary nodules under the guidance of three-dimensional reconstructed computed tomographic angiography. Ann. Thorac. Surg. 89: 877883.

Nouri K, Pinker-Domenig K, Ott J, Fraser I, et al. (2013). Removal of non-palpable Implanon ${ }^{\circledast}$ with the aid of a hook-wire marker. Contraception 88: 577-580.

Oda S, Awai K, Funama Y, Utsunomiya D, et al. (2010). Detection of small pulmonary nodules on chest radiographs: efficacy of dual-energy subtraction technique using flat-panel detector chest radiography. Clin. Radiol. 65: 609-615.

Omori K, Tetsuhara K, Hiramoto H, Shoda H, et al. (2010). A case of primary small intestinal malignant lymphoma found based on multiple pulmonary nodules with cavitation. Nihon Kokyuki Gakkai Zasshi 48: 497-501.

Prosch H, Oschatz E, Eisenhuber E, Wohlschlager H, et al. (2011). CT fluoroscopy guided transpleural cutting needle biopsy of small $(\leq 2.5 \mathrm{~cm})$ subpleural pulmonary nodules. Eur. J. Radiol. 77: 164-166.

Rozen WM, Cham A and Jones T (2010). Managing suspicious cervical lymph nodes after thyroidectomy: the utility of hook-wire needle localization. ANZ J. Surg. 80: 299.

Shan L, Hu J, Li M, Zhang C, et al. (2013). Applications of video-assisted thoracic surgery for the diagnosis and treatment of patients with small pulmonary nodules. Zhongguo Fei Ai Za Zhi 16: 369-372.

Slotty PJ and Stummer W (2012). Comment on: Intra-operative localisation of spinal level using pre-operative CT-guided placement of a flexible hook-wire marker. Br. J. Neurosurg. 26: 785.

Takeda A, Kunieda E, Sanuki N, Aoki Y, et al. (2012). Stereotactic body radiotherapy (SBRT) for solitary pulmonary nodules clinically diagnosed as lung cancer with no pathological confirmation: comparison with non-small-cell lung cancer. Lung Cancer 77: 77-82.

Tamura M, Oda M, Fujimori H, Shimizu Y, et al. (2010). New indication for preoperative marking of small peripheral pulmonary nodules in thoracoscopic surgery. Interact. Cardiovasc. Thorac. Surg. 11: 590-593.

Tsushima K, Sone S, Hanaoka T and Kubo K (2008). Radiological diagnosis of small pulmonary nodules detected on lowdose screening computed tomography. Respirology 13: 817-824.

Yin J, Guo Q, Zhang W, Su H, et al. (2012). Effect of greyscale liquid crystal displays of different resolutions on observer performance during detection of small solitary pulmonary nodules. Br. J. Radiol. 85: 549-555.

Yoshida Y, Inoh S, Murakawa T, Ota S, et al. (2011). Preoperative localization of small peripheral pulmonary nodules by percutaneous marking under computed tomography guidance. Interact. Cardiovasc. Thorac. Surg. 13: 25-28. 